\title{
Purification and characterization of nitrile hydratase of mutant 4D of Rhodococcus rhodochrous PA-34
}

\author{
Amit Pratush $\cdot$ Amit Seth $\cdot$ Tek Chand Bhalla
}

Received: 25 April 2012/Accepted: 18 July 2012/Published online: 24 August 2012

(C) The Author(s) 2012. This article is published with open access at Springerlink.com

\begin{abstract}
Nitrile hydratase (NHase; E.C. 4.2.1.84) has been purified and characterized using ammonium sulfate precipitation, ion exchange chromatography and gel filtration chromatography from the mutant $4 \mathrm{D}$ of Rhodococcus rhodochrous PA-34. The SDS-PAGE and MALDITOF analysis of the purified enzyme revealed that it is dimmer consisting of $\alpha$ - and $\beta$-subunits with a molecular mass of 25 and $30 \mathrm{kDa}$, respectively. The $K_{\mathrm{m}}$ and $V_{\max }$ values were $102 \mathrm{mM}$ and $350.8 \mu \mathrm{mol} / \mathrm{min} / \mathrm{mg}$ using 3 -cyanopyridine as substrate. The purified NHase was stable in higher concentration of potassium ions and in acidic pH 5.5 as compared to NHase of the wild R. rhodochrous PA-34. The analysis of the N-terminal amino acid sequence of this enzyme revealed that this enzyme has $90 \%$ homology with the high molecular weight nitrile hydratase of $R$. rhodochrous $\mathrm{J} 1$.
\end{abstract}

Keywords Characterization · Mutant - Purification · Rhodococcus rhodochrous PA-34

\section{Introduction}

The nitrile metabolism in microorganisms mainly involves nitrilase, nitrile hydratase and amidase enzymes that convert nitriles to amides or acids (Asano et al. 1980; Bhalla et al. 1992; Yamada and Kobayashi 1996). This enzyme is one of the first enzymes used in industry for the production

A. Pratush · A. Seth · T. C. Bhalla $(\square)$

Department of Biotechnology, Himachal Pradesh University, Summer Hill, Shimla 171005, India

e-mail: bhallatc@rediffmail.com of a commodity chemical, i.e., Acrylamide (Raj et al. 2006, 2007; Prasad et al. 2007, 2009). Besides this a number of commercially important products such as nicotinamide, pyrazinamide, thiophenamide etc. have also been synthesized from the nitriles using this enzyme (Raj et al. 2006, 2007; Prasad et al. 2007, 2009; Kobayashi et al. 1992). Yet, there are very few organisms whose nitrile hydratase has shown promise for industrial application (Raj et al. 2006, 2007; Prasad et al. 2007; Kobayashi et al. 1992). The genetically modified organisms or mutants generated through chemical or physical mutagenesis having higher activity have not been either generated or perfected for the large scale applications in the conversion of nitriles to corresponding amides (Pratush et al. 2010).

Rhodococcus rhodochrous PA-34 has been reported as a potential organism that can convert acrylonitrile, butyronitrile and 3-cyanopyridine to corresponding amides at a high concentration (Raj et al. 2006, 2007; Prasad et al. 2007; Bhalla and Kumar 2005). A mutant 4D has been generated by chemical mutagenesis of $R$. rhodochrous PA34 using MNNG that exhibited twofold increase in its nitrile hydratase activity for the conversion of 3-cyanopyridine to nicotinamide (Pratush et al. 2010). In this communication, we report the purification and characterization of nitrile hydratase of mutant 4D of $R$. rhodochrous PA-34.

\section{Materials and methods}

Chemicals

Nicotinamide and 3-cyanopyridine used in the present study were purchased from Sigma and Alfa Aesar, respectively. Other chemicals used in the present study were of analytical grade from various commercial sources. 
Microorganism

The mutant 4D of $R$. rhodochrous PA-34 generated earlier by Pratush et al. (2010) at the Department of Biotechnology, Himachal Pradesh University, Shimla, India.

Culture, nitrile hydratase assay and estimation of nicotinamide

Mutant 4D cells were grown for the production of nitrile hydratase by following the procedure as detailed by Pratush et al. (2010). The NHase activity was assayed in $1 \mathrm{ml}$ reaction containing $880 \mu \mathrm{l}, 0.3 \mathrm{M}$ Potassium phosphate buffer (pH 5.5), $20 \mu \mathrm{l}(0.08 \mathrm{mg} / \mathrm{ml})$ of enzyme and $100 \mu \mathrm{l}$ of substrate $0.5 \mathrm{M} 3$-cyanopyridine following the method reported previously (Prasad et al. 2004).

Purification of nitrile hydratase of mutant strain

All steps of purification were performed at $4{ }^{\circ} \mathrm{C}$ and $0.3 \mathrm{M}$ potassium phosphate buffer $\mathrm{pH} 5.5$ at $4{ }^{\circ} \mathrm{C}$ was used.

1. Homogenization of resting cells of $R$. rhodochrous PA-34 mutant 4D

The resting cells of mutant 4D were disrupted using bead beater (BSP make) having Zirconium beads $(0.1 \mathrm{~mm}$ diameter) for $30 \mathrm{~min}$ in 10 disruption cycles at $4{ }^{\circ} \mathrm{C}$. The resulting cell-free extract (CFE) was used as crude enzyme for subsequent NHase purification.

\section{Ammonium sulfate fractionation of $\mathrm{CFE}$}

The cell-free extract was subjected to various \% saturation concentration of ammonium sulfate $(0-80 \%)$. The fraction exhibiting maximum activity of NHase was termed as ASF and was taken for further purification of NHase.

\section{Gel filtration of ASF}

Ammonium sulfate fractionation was filtered through $0.45-\mu \mathrm{m}$ filter and directly loaded on to pre-packed Sephacryl S-300 gel filtration column (16 mm diameter $\times 600 \mathrm{~mm}$ length) equilibrated with buffer. The gel filtration chromatography was performed using AKTA prime $^{\mathrm{TM}} \mathrm{V} 2.00$ at a flow rate $0.3 \mathrm{ml} / \mathrm{min}$ of elution buffer (0.3 M potassium phosphate buffer $\mathrm{pH} 5.5$ ). NHase active fractions were subjected to SDS polyacrylamide gel electrophoresis (PAGE). The fractions exhibiting NHase activity were pooled. These pooled fractions were termed as GFF and used for further purification of NHase.

\section{DEAE-ion exchange chromatography of GFF}

The pooled filtered fractions of gel filtration (GFF) applied on an DEAE to an ion-exchange chromatography column $(16 \mathrm{~mm}$ diameter $\times 100 \mathrm{~mm}$ length $)$ equilibrated with $0.3 \mathrm{M}$ potassium phosphate buffer $\mathrm{pH}$ 5.5. The column was eluted with a linear gradient of $\mathrm{NaCl}$ from 0.1 to $0.7 \mathrm{M}$ in $0.3 \mathrm{M}$ potassium phosphate buffer. NHase activity and protein concentration were estimated in each fraction. The fractions exhibiting single band on nativePAGE were pooled and termed as DEAEF (i.e., purified NHase).

Characterization of purified NHase

Buffer, pH, temperature, substrate specificity and effect of metal ions and inhibitors on activity of purified NHase of mutant $4 D$

Various buffer systems (such as sodium phosphate and Tris- $\mathrm{HCl}$ and potassium phosphate buffer each $0.1 \mathrm{M} \mathrm{pH}$ 7.2) were used to select a suitable buffer to assay the activity of the purified NHase. The ionic strength of buffer (0.1-0.5 M potassium phosphate buffer) and $\mathrm{pH}(5.0,5.5$, $6.0,6.5,7.0,7.5,8.0$, and 8.5) of selected buffer (potassium phosphate buffer) were optimized for NHase assay.

To determine temperature optimum of NHase of mutant $4 \mathrm{D}$, the activity of this enzyme was determined at $10-80{ }^{\circ} \mathrm{C}$. Thermal stability and substrate specificity profile of the purified mutant 4D NHase were tested after an interval of $20 \mathrm{~min}$ by subjecting purified NHase to 45, 55 and $65^{\circ} \mathrm{C}$ for $8 \mathrm{~h}$ with different substrates, i.e., 2-cyanopyridine, 3-cyanopyridine, 4-cyanopyridine, butyronitrile, benzonitrile and acrylonitrile, respectively.

The effects of metal ions $\left(\mathrm{AgNO}_{3}, \mathrm{CaCl}_{2}, \mathrm{CdCl}_{2}, \mathrm{CoCl}_{2}\right.$, $\mathrm{CuCl}_{2}, \mathrm{FeCl}_{2}, \mathrm{HgCl}_{2}, \mathrm{MgCl}_{2}$ and $\mathrm{MnCl}_{2}$ ) and inhibitors (ammonium persulfate (APS), DTT, EDTA, hydroxylamine, iodoacetic acid, L-ascorbic acid, phenyl hydrazine, phenylmethanesulphonylfluoride, semicarbazide, sodium azide and urea) on NHase activity of mutant were investigated by pre-incubating the enzyme at $1 \mathrm{mM}$ concentration of metal ions/inhibitors for $30 \mathrm{~min}$ at $55^{\circ} \mathrm{C}$ and then the NHase activity was assayed.

Determination of $K_{\mathrm{m}}$ and $V_{\max }$ of NHase

The $K_{\mathrm{m}}$ and $V_{\max }$ of purified NHase of mutant 4D was calculated by determining initial velocity $(v)$ of NHase at various concentrations of 3-cyanopyridine.

Determination of molecular mass of NHase N-terminal amino acid sequencing and MALDI-TOF analysis of purified NHase of $R$. rhodochrous PA-34 mutant 4D and its analysis

SDS and native-PAGE were carried out to determine the purity, molecular mass of NHase and its subunits by the method of Laemmli (1970). The N-terminal amino acid 
sequence of purified NHase of mutant 4D was done at the Institute of Microbial Technology (IMTECH), Sector 39A, Chandigarh (India). Matrix assisted laser dissociated ionization-time of flight (MALDI-TOF) analysis of purified NHase was done at Jawaharlal Nehru University, New Delhi (India). Multiple protein sequence alignment was carried out using Clustal W program (Thompson et al. 1997; Chenna et al. 2003).

\section{Results and discussion}

Purification of mutant 4D NHase

The disruption of $2.0 \mathrm{~g}$ of resting cells of mutant 4D (containing 11,240 $\mathrm{U}$ of NHase activity) released $119 \mathrm{mg}$ of protein and 1,000 $\mathrm{U}$ NHase activity in CFE. The purification of NHase of mutant 4D from the CFE involved ammonium sulfate fractionation (ASF), gel filtration chromatography (GFF) on Sephacryl S-300, DEAE-ion
Table 1 Purification table of nitrile hydratase of mutant

\begin{tabular}{llllll}
\hline $\begin{array}{l}\text { Stages of } \\
\text { purifications }\end{array}$ & $\begin{array}{l}\text { Total } \\
\text { protein } \\
\text { (mg) }\end{array}$ & $\begin{array}{l}\text { Specific } \\
\text { activity } \\
\text { (units) }\end{array}$ & $\begin{array}{l}\text { Total } \\
\text { activity } \\
\text { (units) }\end{array}$ & $\begin{array}{l}\text { Yield } \\
(\%)\end{array}$ & $\begin{array}{l}\text { Purification } \\
\text { (fold) }\end{array}$ \\
\hline $\begin{array}{l}\text { Crude sample } \\
\text { (CFE) }\end{array}$ & 119 & 8.4 & 1,000 & 100 & - \\
$\begin{array}{l}\text { Ammonium } \\
\text { sulfate } \\
\text { precipitation } \\
\text { (ASF) }\end{array}$ & 90.7 & 9.2 & 835 & 83 & 1.0 \\
$\begin{array}{l}\text { Gel filtration (S- } \\
\text { 300) (GFF) }\end{array}$ & 45.4 & 15.2 & 690 & 82 & 1.7 \\
$\begin{array}{l}\text { DEAE-ion } \\
\text { exchange }\end{array}$ & 9.6 & 45 & 432 & 62 & 3 \\
(DEAEF) & & & & & \\
\hline
\end{tabular}

exchange chromatography (DEAEF). NHase protein was precipitated at 30-40\% saturation of ammonium sulfate (ASF), and it contained $90.7 \mathrm{mg}$ protein with specific activity of $9.2 \mathrm{U} / \mathrm{mg}$ proteins (Table 1). Ammonium sulfate fractionation (30-40\% saturation cut) resulted in
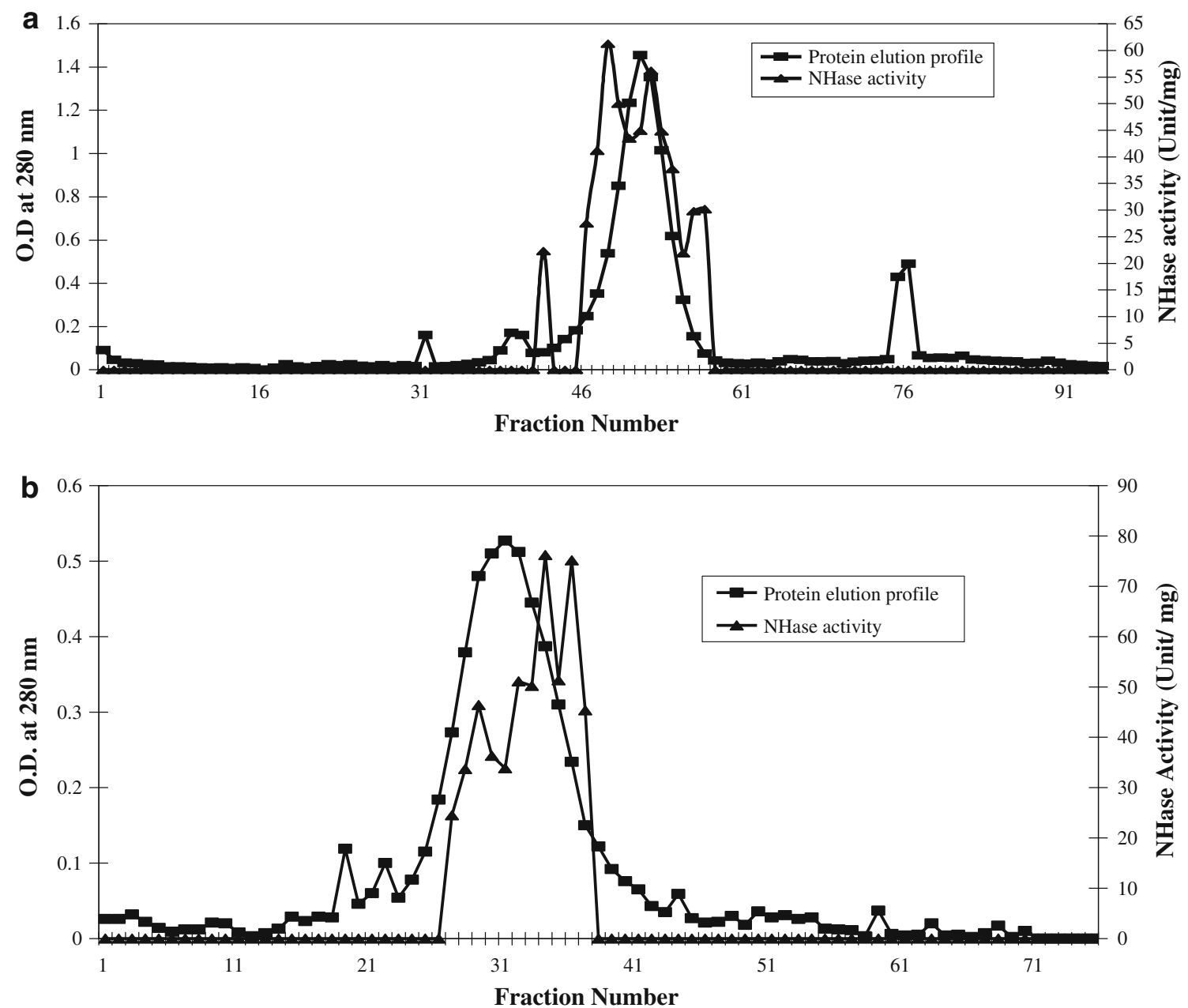

Fig. 1 a Protein and NHase activity profile during gel permeation chromatography. b Protein and NHase activity profile by DEAE ion exchange chromatography 


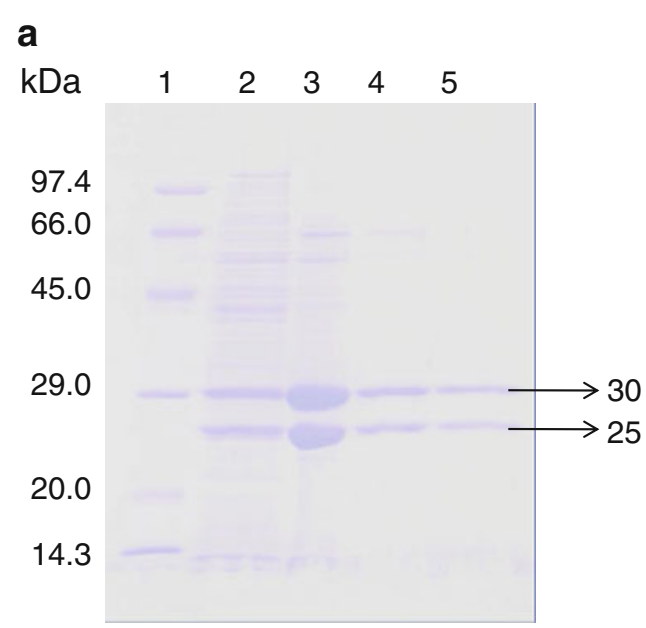

Fig. 2 a Sodium dodecyl sulfate-polyacrylamide gel electrophoresis of NHase of mutant 4D at various steps of purification. SDS-protein molecular weight markers in $\mathrm{kDa}=$ kilo Dalton (lane 1), cell-free protein extract (lane 2), ammonium sulfate precipitation sample (lane 3), gel filtration chromatography samples (lane 4) and DEAE-ion exchange chromatography sample (lane 5) SDS PAGE of purified NHase of mutant. b Native-PAGE analysis of purified NHase of

onefold purification of enzyme (AFS) with an yield of $83 \%$ of NHase activity (Table 1). The NHases of R. rhodochrous PA-34 (Prasad et al. 2009), R. rhodochrous J1 (low and high molecular weight both) (Wieser et al. 1998; Nagasawa et al. 1991) and Rhodococcus sp. strain YH3-3 (Kato et al. 1999), Brevibacterium R312 (Nagasawa et al. 1986) and Pseudomonas chlororaphis B23 (Nagasawa et al. 1987) were precipitated in the 30-70\% and 40-55\% cut off ammonium sulfate, respectively.

The dialysed protein of ASF was subjected to gel permeation chromatography using S-300 column (Fig. 1a). This step resulted in 1.7 -fold of purification with an yield of $82 \%$ having specific NHase activity of 15.2 U/mg protein. The fraction of GFF reached in NHase proteins was further loaded to the DEAE-ion exchange column (Fig. 1b), which resulted in the threefold purification of enzyme with a yield of $62 \%$ and specific activity of $45 \mathrm{U} / \mathrm{mg}$ protein (Table 1). Earlier, the NHase of $R$. rhodochrous $\mathrm{J} 1$, Agrobacterium tumefaciens strain d3, B. pallidus Dac521, P. chlororaphis B23 and Brevibacterium R312 were purified by employing gel permeation chromatography techniques (Wieser et al. 1998; Nagasawa et al. 1986, 1987; Bauer et al. 1998; Cramp and Cowan 1999).

\section{Characterization of NHase of mutant 4D}

The purified NHase consisted of two polypeptides one comprising $25 \mathrm{kDa}$ ( $\alpha$-subunit) and other was of $30.6 \mathrm{kDa}$ ( $\beta$-subunit) (Fig. 2a). It means that this enzyme is also constituted by two different polypeptides $(\alpha-$ and $\beta$ - b

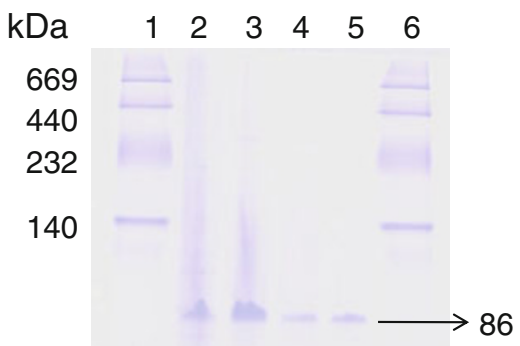

66 mutant 4D, lane 1 was loaded with following molecular mass standards: thyroglobulin $(660 \mathrm{kDa})$, ferritin $(440 \mathrm{kDa})$, catalase $(232 \mathrm{kDa})$, lactate dehydrogenase $(140 \mathrm{kDa})$ and albumin (66 $\mathrm{kDa}$ ). Cell-free extract (lane 2), ammonium sulfate fraction (lane 3) and gel permeation column chromatography fraction (lane 4) DEAEion exchange column chromatography fraction (lane 5)

subunits) similar to the earlier reported NHases (Prasad et al. 2009; Wieser et al. 1998; Nagasawa et al. 1986). Native PAGE of purified NHase revealed a single band of $86 \mathrm{kDa}$ (Fig. 2b). This indicated that the functional NHase might be constituted by one $\alpha$ - and two $\beta$-subunits (i.e., $\alpha \beta_{2}$ ). The molecular mass of nitrile hydrates of different organisms varies from species to species, i.e., $R$. rhodochrous $\mathrm{J} 1, R$. rhodochrous sp. N774 and Cornybacterium sp. C5 have 59, 70 and $61 \mathrm{kDa}$, respectively (Kobayashi et al. 1991; Endo and Watanabe 1989; Yamamoto et al. 1992). The $K_{\mathrm{m}}$ and $V_{\max }$ values for the purified NHase of mutant 4D were $102 \mathrm{mM}$ and $350.8 \mu \mathrm{mol} / \mathrm{min} / \mathrm{mg}$, respectively, using 3-cyanopyridine as substrate (Fig. 3), whereas $K_{\mathrm{m}}$ and $V_{\max }$ reported from other sources such as $R$. rhodochrous $\mathrm{PA}-34, \mathrm{~L}$ and $\mathrm{H}-\mathrm{NH}$ ase of $R$. rhodochrous $\mathrm{J} 1$ were $167 \mathrm{mM}$ and $250 \mu \mathrm{mol} / \mathrm{min} / \mathrm{mg}, 0.30 \mathrm{mM}$ and $579 \mu \mathrm{mol} / \mathrm{min} / \mathrm{mg}$ and $200 \mathrm{mM}$ and $370 \mu \mathrm{mol} / \mathrm{min} / \mathrm{mg}$ using 3-cyanopyridine as substrate, respectively (Prasad et al. 2009; Wieser et al. 1998). These results indicated that

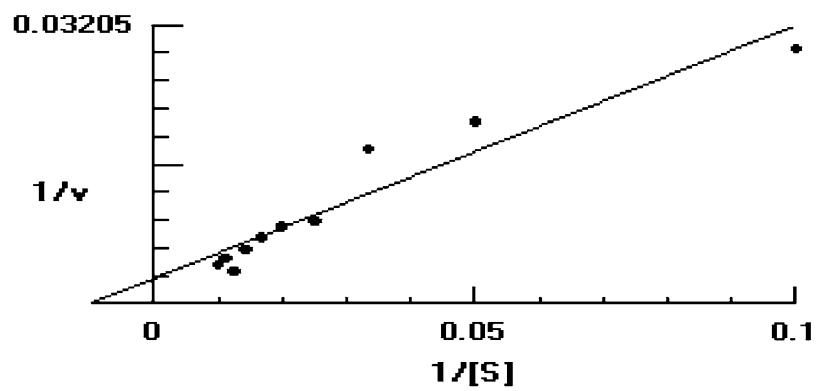

Fig. 3 Lineweaver Burk plot of purified NHase of mutant 

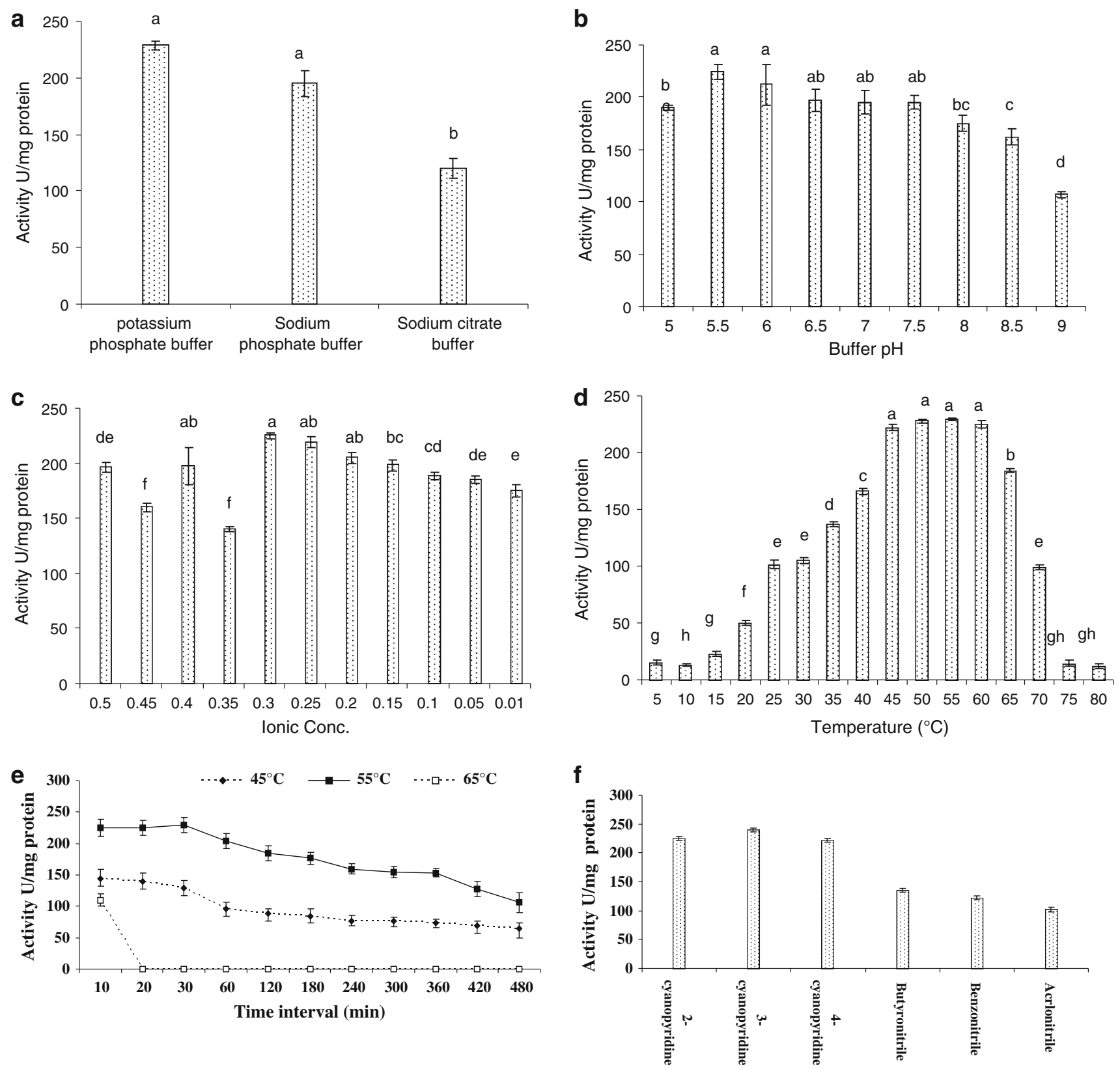

Fig. 4 a Buffer system optimization for purified NHase of mutant. b Ionic strength optimization of buffer for purified NHase of mutant. c Buffer $\mathrm{pH}$ optimization for purified NHase of mutant. d Reaction

temperature optimization for purified NHase of mutant. e Thermostability profile of purified NHase of mutant at 45,55 and $65{ }^{\circ} \mathrm{C}$. f Effect of different substrates on purified NHase of mutant

this enzyme had higher affinity for 3-cyanopyridine and had higher $V_{\max }$ in comparison to its wild strain. Among the three types of buffers tested, maximum NHase activity (225 $\mathrm{U})$ was rerecorded in $0.3 \mathrm{M}$ potassium phosphate buffer (Fig. 4a). Below and above $0.3 \mathrm{M}$ concentration of the buffer the activity of NHase drastically decreased (Fig. 4b). The mutant NHase was found to be stable in acidic conditions, i.e., pH 5.5 (Fig. 4c). These results are drastically different from the earlier reports of Banerjee et al. (2002). Moreover, this NHase exhibited higher

concentration of potassium ions, i.e., $0.3 \mathrm{M}$ (Fig. 4b), whereas other reported NHases showed higher activity at $0.1 \mathrm{M}$ potassium phosphate buffer (Raj et al. 2006; Prasad et al. 2009). The optimum temperature for assay of NHase activity turns out to be $55^{\circ} \mathrm{C}$ (Fig. $4 \mathrm{~d}$ ), whereas it is $40{ }^{\circ} \mathrm{C}$ for $R$. rhodochrous PA-34 and $R$. rhodochrous $\mathrm{J} 1$ (Prasad et al. 2009; Wieser et al. 1998). Most of the nitrile hydratases have exhibited maximum activity near ambient temperature between 20 and $35{ }^{\circ} \mathrm{C}$ (Banerjee et al. 2002). The thermophilic NHases of Bacillus RAPc8 and 
Pseudonocardia thermophila showed maximum activity at $60{ }^{\circ} \mathrm{C}$ (Yamaki et al. 1997; Pereira et al. 1998).

The NHase of mutant 4D showed its maximum activity at $55{ }^{\circ} \mathrm{C}$ and it remained stable up to $5 \mathrm{~h}$ at this temperature, whereas the enzyme completely lost its activity at $65^{\circ} \mathrm{C}$ (Fig. 4e). However, a thermophilic NHase from $B$. pallidus Dac 521 had a half-life of $0.85 \mathrm{~h}$ at $50{ }^{\circ} \mathrm{C}$. The NHase from mutant 4D was much more stable than earlier reported NHase at higher temperature (Banerjee et al. 2002). NHase of mutant 4D showed highest activity with 3-cyanopyridine (Fig. 4f). The purified NHase of mutant $4 \mathrm{D}$, showed almost complete loss of activity in the presence of metal ions. The NHase activity was inhibited up to 65.5-73.7 \% by $\mathrm{MgCl}_{2}$ and hydroxyl amine, respectively. An inhibition of $30.5 \%$ in NHase activity was observed in the presence of PMSF in reaction (Table 2). The loss of activity of NHase of mutant 4D in the presence of metal

Table 2 Effect of metal ions and compounds on purified nitrile hydratase of mutant

\begin{tabular}{ll}
\hline Metal ion/inhibitor $(1 \mathrm{mM})$ & Relative activity $(\%)$ \\
\hline None & 100 \\
$\mathrm{AgNO}_{3}$ & 0.266 \\
$\mathrm{CaCl}_{2}$ & 77 \\
$\mathrm{CdCl}_{2}$ & 61.8 \\
$\mathrm{CoCl}_{2}$ & 74.8 \\
$\mathrm{CuCl}_{2}$ & 0.824 \\
$\mathrm{FeCl}_{2}$ & 0.74 \\
$\mathrm{HgCl}_{2}$ & 0.04 \\
$\mathrm{MgCl}_{2}$ & 34.5 \\
$\mathrm{MnCl}_{2}$ & 73.5 \\
Ammonium persulfate & 42.5 \\
Dithiothreitol & 57.13 \\
EDTA & 71.3 \\
Hydroxylamine & 26.3 \\
Iodoacetic acid & 71.23 \\
L-Ascorbic acid & 72.6 \\
Phenyl hydrazine & 8.0 \\
PMSF & 69.5 \\
Sodium azide & 77.3 \\
Urea & 83.8 \\
\hline
\end{tabular}

Table 3 N-terminal sequence of $\beta$ - and $\alpha$-subunits of mutant $4 \mathrm{D}$ and wild Rhodococcus rhodochrous PA-34

\begin{tabular}{ll}
\hline Subunit name & N-terminal sequence \\
\hline$\beta$-subunit of mutant & M D G F H D T G N M \\
$\beta$-subunit of wild & M D G I H D L G G R \\
$\alpha$-subunit of mutant & T E H V N K Y T E A \\
$\alpha$-subunit of wild & T A H N P V Q G K L \\
\hline
\end{tabular}

ions indicated the involvement of Cys residue at its active site, which might have got complexed with metal ions leading to inactivation of the enzyme.

The N-terminal sequence of $\beta$ - and $\alpha$-subunits of mutant 4D NHase showed a significant (94\%) change in $\alpha$-subunit when compared with the $\mathrm{N}$-terminal sequence of $\alpha$-subunit of wild $R$. rhodochrous PA-34, whereas the N-terminal sequence of $\beta$-subunit of mutant showed $90 \%$ homology with the $\beta$-subunit of wild (Table 3). MALDI-TOF analysis revealed that purified NHase of mutant 4D had $90 \%$ homology with the high molecular weight NHase of $R$. rhodochrous J1 (Kobayashi et al. 1991). The N-terminal and MALDI-TOF analysis of mutant 4D NHase protein revealed a significant change in the amino acid sequence of $\alpha$ - and $\beta$-subunits of wild strain and it exhibited high homology with H-NHase reported earlier from $R$. rhodochrous J1 (Kobayashi et al. 1991). These studies suggest that the wild strain $R$. rhodochrous PA-34 produces the L-NHase (low molecular weight nitrile hydratase), whereas the $R$. rhodochrous PA-34 mutant 4D produces H-NHase generated through chemical mutagenesis using MNNG. The chemical mutagenesis has switched off the expression of L-NHase gene and switch on the expression of H-NHase gene. However, further studies on cloning and sequencing of NHase operon of the wild and mutant 4D strains of $R$. rhodochrous PA-34 are needed further to explore this aspect at molecular level.

\section{Conclusion}

The NHase of $R$. rhodochrous PA-34 of mutant 4D produces high molecular nitrile hydratase with better thermal, ionic and acidic $\mathrm{pH}$ stabilities as compared to earlier reported nitrile hydratases including the NHase of wild strain.

Acknowledgments The research work was supported by a research grant from University Grants Commission, Government of India, New Delhi, India. The authors also wish to acknowledge the internet facilities provided by Sub-Distributed Information centre, Department of Biotechnology, Himachal Pradesh University, Shimla-5, India.

Open Access This article is distributed under the terms of the Creative Commons Attribution License which permits any use, distribution, and reproduction in any medium, provided the original author(s) and the source are credited.

\section{References}

Asano Y, Tani Y, Yamada H (1980) A new enzyme 'nitrile hydratase' which degrades acetonitrile in combination with amidase. Agri Biol Chem 44:2251-2252

Banerjee A, Sharma R, Banerjee UC (2002) The nitrile-degrading enzymes: current status and future prospects. Appl Microbiol Biotechnol 60:33-44 
Bauer R, Knackmuss HJ, Stolz A (1998) Enantioselective hydration of 2-arylpropionitriles by a nitrile hydratase from Agrobacterium tumefaciens strain d 3. Appl Microbiol Biotechnol 49:89-95

Bhalla TC, Kumar H (2005) Nocardia globerula NHB-2: a versatile nitrile-degrading organism. Can J Microbiol 51:705-708

Bhalla TC, Miura M, Wakamoto A, Ohba Y, Furuhashi K (1992) Asymmetric hydrolysis of $\alpha$-aminonitriles to optically active amino acids by a nitrilase of Rhodococcus rhodochrous PA-34. Appl Microbiol Biotechnol 37:184-190

Chenna R, Sugawara H, Koike T, Lopez R, Gibson TJ, Higgins DG, Thompson JD (2003) Multiple sequence alignment with the Clustal series of programs. Nucleic Acids Res 31:3497-3500

Cramp RA, Cowan DA (1999) Molecular characterisation of a novel thermophilic nitrile hydratase. Biochem Biophys Acta (BBA) Protein Struct Mol Enzymol 1431:249-260

Endo T, Watanabe I (1989) Nitrile hydratase of Rhodococcus sp. $\mathrm{N}-774$ purification and amino acid sequences. FEBS Lett 243:61-64

Kato Y, Tsuda T, Asano Y (1999) Nitrile hydratase involved in aldoxime metabolism from Rhodococcus sp. strain YH3-3 purification and characterization. Eur J Biochem 263:662-670

Kobayashi M, Nishiyama M, Nagasawa T, Horinouchi S, Beppu T, Yamada H (1991) Cloning, nucleotide sequence and expression in Escherichia coli of two cobalt-containing nitrile hydratase genes from Rhodococcus rhodochrous J1. Biochem Biophys Acta 1129:23-33

Kobayashi M, Nagasawa T, Yamada H (1992) Enzymatic synthesis of acrylamide: a success story not yet over. Trends Biotechnol 10:402-408

Laemmli UK (1970) Cleavage of structural proteins during the assembly of the head of bacteriophage T4. Nature 227:680-685

Nagasawa T, Ryuno K, Yamada H (1986) Nitrile hydratase of Brevibacterium R312-purification and characterization. Biochem Biophys Res Comm 139:1304-1312

Nagasawa T, Nanba H, Ryuno K, Takeuchi K, Yamada H (1987) Nitrile hydratase of Pseudomonas chlororaphis B23-purification and characterization. Eur J Biochem 162:691-698

Nagasawa T, Takechi K, Nardidei V, Mihara Y, Yamada H (1991) Optimum culture conditions for the production of cobaltcontaining nitrile hydratase by Rhodococcus rhodochrous $\mathrm{J} 1$. Appl Microbiol Biotechnol 34:783-788
Pereira RA, Graham D, Rainey FA, Cowan DA (1998) A novel thermostable nitrile hydratase. Extremophiles 2:347-357

Prasad S, Raj J, Bhalla TC (2004) Optimization of culture conditions for hyper production of nitrile hydratase of Rhodococcus rhodochrous PA-34. Indian J Microbiol 44:251-256

Prasad S, Raj J, Bhalla TC (2007) Bench scale conversion of 3-cyanopyridine to nicotinamide using resting cells of Rhodococcus rhodochrous PA-34. Indian J Microbiol 47:34-41

Prasad S, Raj J, Bhalla TC (2009) Purification of a hyperactive nitrile hydratase from resting cells of Rhodococcus rhodochrous PA34. Indian J Microbiol 49:237-242

Pratush A, Seth A, Bhalla TC (2010) Generation of mutant of Rhodococcus rhodochrous PA-34 through chemical mutagenesis for hyperproduction of nitrile hydratase. Acta Microbiol Immun Hungarica 57(2):135-146

Raj J, Prasad S, Bhalla TC (2006) Rhodococcus rhodochrous PA-34 a potential catalyst for acrylamide synthesis. Process Biochem 41(6): 1359-1363

Raj J, Seth A, Prasad S, Bhalla TC (2007) Bioconversion of butyronitrile to butyramide using whole cells of Rhodococcus rhodochrous PA-34. Appl Microbiol Biotechnol 74:535-539

Thompson JD, Gibson TJ, Plewniak F, Jeanmougin F, Higgins DG (1997) The ClustalX windows interface: flexible strategies for multiple sequence alignment aided by quality analysis tools. Nucleic Acids Res 25:4876-4882

Wieser M, Takeuchi K, Wada Y, Yamada H, Nagasawa T (1998) Low-molecular weight nitrile hydratase from Rhodococcus rhodochrous $\mathrm{J} 1$ : purification, substrate specificity and comparison with the analogous high-molecular-mass enzyme. FEMS Microbiol Lett 169:17-22

Yamada H, Kobayashi M (1996) Nitrile hydratase and its application to industrial production of acrylamide. Biosci Biotechnol Biochem 60:1391-1400

Yamaki T, Oikawa T, Ito K, Nakamura T (1997) Cloning and sequencing of a nitrile hydratase gene from Pseudonocardia thermophila JCM 3095. J Ferment Bioeng 83:474-477

Yamamoto K, Fujimatsu I, Komatsu KI (1992) Purification and characterization of the nitrilase from Alcaligenes faecalis ATCC 8750 responsible for enantioselective hydrolysis of mandelonitrile. J Ferment Bioeng 73:425-430 